\title{
On the sum of the two largest Laplacian eigenvalues of trees
}

\author{
Mei Guan ${ }^{1}$, Mingqing Zhai ${ }^{2 *}$ and Yongfeng $\mathrm{Wu}^{3}$
}

${ }^{\text {*Correspondence: }}$

mqzhai@chzu.edu.cn

${ }^{2}$ School of Mathematical Science,

Chuzhou University, Chuzhou,

239012, China

Full list of author information is

available at the end of the article

\begin{abstract}
For $S(T)$, the sum of the two largest Laplacian eigenvalues of a tree $T$, an upper bound is obtained. Moreover, among all trees with $n \geq 4$ vertices, the unique tree which attains the maximal value of $S(T)$ is determined.

MSC: $05 C 50$

Keywords: upper bound; tree; Laplacian matrix
\end{abstract}

\section{Introduction}

Let $V(G)$ be the vertex set and $E(G)$ be the edge set of a graph $G$. The numbers of vertices and edges of $G$ are denoted by $n(G)$ and $m(G)$, respectively. For a vertex $v \in V(G)$, let $N_{G}(v)$ be the set of vertices adjacent to $v$ and $d_{G}(v)=\left|N_{G}(v)\right|$ be the degree of $v$. Particularly, denote by $\Delta(G)$ the maximum degree of $G$. The diameter of a connected graph $G$, denoted by $d(G)$, is the maximum distance among all pairs of vertices in $G$. Let $A(G)$ be the adjacency matrix of $G$ and $D(G)$ be the diagonal matrix of vertex degrees. The matrix $D(G)-A(G)$ is called the Laplacian matrix of $G$ and its eigenvalues are called the Laplacian eigenvalues of $G$. Let $\mu_{1} \geq \mu_{2} \geq \cdots \geq \mu_{n}$ be the Laplacian eigenvalues of a graph $G$ with $n$ vertices. It is well known that $\mu_{n}=0$ and $\sum_{i=1}^{n-1} \mu_{i}=2 m(G)$. In particular, $\mu_{n-1}$ is called the algebraic connectivity of $G$ and it is denoted by $\alpha(G)$.

The Laplacian matrix is an important topic in the theory of graph spectra. Particularly, much literature has paid attention to $\mu_{1}, \mu_{2}, \mu_{n-1}$ or $\mu_{1}-\mu_{n-1}$ for trees (see, for example, [1-6]). Let $S_{n}$ be the star of order $n, S_{a, b}^{k}$ be the tree obtained from two stars $S_{a+1}, S_{b+1}$ by joining a path of length $k$ between their central vertices (see Figure 1). As is well known, among all trees of order $n, S_{n}$ has the largest value of $\mu_{1}$ (see [7]) and $S_{n-3,1}^{1}$ has the second largest value of $\mu_{1}$ (see [6]). On the other hand, Guo [4] proved that these two trees also attain the first two smallest values of $\mu_{2}$, respectively. This implies that $\mu_{1}, \mu_{2}$ cannot attain simultaneously the maximal (or minimal) value and even the relation between them seems like a seesaw. Therefore, it is interesting to investigate the value of $\mu_{1}+\mu_{2}$. Moreover, Zhang [6] showed that the $S_{k-1, k-1}^{1}, S_{k-1, k-2}^{2}, S_{k-2, k-2}^{3}$ attain simultaneously the largest value of $\mu_{2}$ among all trees with $2 k$ vertices. Then Shao et al. [5] showed that $S_{k-1, k-1}^{2}$ attains the largest value of $\mu_{2}$ among all trees with $2 k+1$ vertices.

Another motivation to study the value of $\mu_{1}+\mu_{2}$ came from a result of Haemers et al. [8], who showed that $\mu_{1}+\mu_{2} \leq m(G)+3$ for any graph $G$. This result implies that Brouwer's 
Figure $1 S_{a, b}^{k}$ : a tree of order $a+b+k+1$.

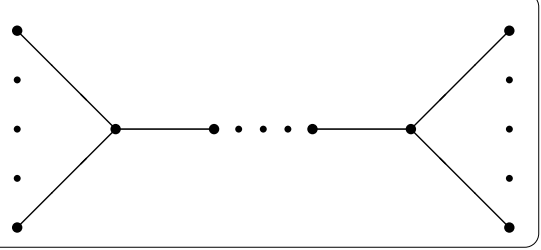

conjecture [9],

$$
\mu_{1}+\mu_{2}+\cdots+\mu_{k} \leq m(G)+\left(\begin{array}{c}
k+1 \\
2
\end{array}\right)
$$

is true for $k=2$. Considering a tree $T$, we have $\mu_{1}+\mu_{2} \leq n(T)+2$. Recently, Fritscher et al. [10] improved this bound by giving $\mu_{1}+\mu_{2}<n(T)+2-\frac{2}{n(T)}$. This paper determines the extremal tree that attains the bound of $\mu_{1}+\mu_{2}$. Moreover, for general connected graphs, we also give a conjecture on the extremal graphs for $\mu_{1}+\mu_{2}$.

\section{A sharp upper bound of $\mu_{1}+\mu_{2}$}

Let $S_{k}(G)$ be the sum of the largest $k$ Laplacian eigenvalues of a graph $G$. When $k=2$, we shall write $S(G)$ instead of $S_{k}(G)$ for simplicity. For graphs $G$ and $H$, we denote by $G \cup H$ the graph with vertex set $V(G) \cup V(H)$ and edge set $E(G) \cup E(H)$. The following lemmas come from an important result as regards a real symmetric matrix.

Lemma 2.1 ([8]) Let $G_{1}, G_{2}, \ldots, G_{r}$ be some edge-disjoint graphs. Then $S_{k}\left(\bigcup_{i=1}^{r} G_{i}\right) \leq$ $\sum_{i=1}^{r} S_{k}\left(G_{i}\right)$ for any $k$.

Lemma 2.2 ([8]) For any graph $G, S(G) \leq m(G)+3$.

Lemma 2.3 Let $G$ be a connected graph, $d_{i}=d_{G}\left(v_{i}\right)$ and $m_{i}=\Sigma_{v_{j} \in N_{G}\left(v_{i}\right)} d_{j} / d_{i}$. Then

(i) [11] $\mu_{1}(G) \geq \Delta(G)+1$, with equality if and only if $\Delta(G)=n(G)-1$.

(ii) [7] $\mu_{1}(G) \leq n(G)$, with equality if and only if the complement of $G$ is disconnected.

(iii) [12] $\mu_{1}(G) \leq \max \left\{d_{i}+m_{i} \mid v_{i} \in V(G)\right\}$.

Lemma 2.4 ([6]) Let $T$ be a tree oforder $n$. If $T ¥ S_{n}$, then $\mu_{1}(T) \leq \mu_{1}\left(S_{n-3,1}^{1}\right)$, with equality if and only if $T \cong S_{n-3,1}^{1}$.

Corollary 2.5 Let $T$ be a tree with $n$ vertices and diameter $d \geq 3$. Then $\mu_{1}(T)<n-0.5$.

Proof Note that any tree $T$ has diameter $d \geq 3$ if $T ¥ S_{n}$. According to Lemma 2.4, $\mu_{1}(T) \leq$ $\mu_{1}\left(S_{n-3,1}^{1}\right)$. Further, by Lemma 2.3,

$$
\mu_{1}\left(S_{n-3,1}^{1}\right) \leq \max \left\{d_{i}+m_{i}\right\}=n-2+\frac{n-1}{n-2}=n-1+\frac{1}{n-2}<n-0.5
$$

for $n \geq 5$. For $n=4$, a straightforward calculation shows that $\mu_{1}\left(S_{1,1}^{1}\right)=2+\sqrt{2}<3.5$.

Lemma 2.6 ([2]) Let $T$ be a tree of order $n$ and diameter $d \geq 3$. Then $\alpha(T) \geq$ $\alpha\left(S_{\left\lceil\frac{n-d+1}{2}\right\rceil,\left\lfloor\frac{n-d+1}{2}\right\rfloor}^{d-2}\right)$, with equality if and only if $T \cong S_{\left\lceil\frac{n-d+1}{2}\right\rceil,\left\lfloor\frac{n-d+1}{2}\right\rfloor}^{d-2}$. 
Lemma 2.7 ([11]) Let $G$ be a graph with a vertex $u$ of degree one. Then $\alpha(G) \leq \alpha(G-u)$.

Lemma 2.7 implies that the algebraic connectivity of a tree is not greater than that of its subtree.

Lemma 2.8 ([4]) Let $T_{n}^{k}(n \geq 2 k+1)$ be a tree obtained from a star $S_{n-k}$ by replacing its $k$ edges with $k$ paths of length two, respectively. If $k \geq 2$, then $\mu_{2}\left(T_{n}^{k}\right)=\frac{3+\sqrt{5}}{2}$.

The following lemma can be found in [13] and is known as the Interlacing Theorem of Laplacian eigenvalues.

Lemma 2.9 Let $G$ be a graph of order $n$ and $H$ be a graph obtained from $G$ by deleting an edge. Then

$$
\mu_{1}(G) \geq \mu_{1}(H) \geq \cdots \geq \mu_{n}(G) \geq \mu_{n}(H)=0 .
$$

Next we give the main theorem of this section. Its proof is divided into several sequent claims.

Theorem 2.10 For any tree $T$ with order $n \geq 4, S(T) \leq S\left(S_{\left\lceil\frac{n-2}{2}\right\rceil,\left\lfloor\frac{n-2}{2}\right\rfloor}^{1}\right)$. The equality holds if and only if $T \cong S_{\left\lceil\frac{n-2}{2}\right\rceil,\left\lfloor\frac{n-2}{2}\right\rfloor}^{1}$.

Claim 2.11 For any tree $T$ with order $n \geq 4$ and diameter $d \leq 3, S(T)<S\left(S_{\left\lceil\frac{n-2}{2}\right\rceil,\left\lfloor\frac{n-2}{2}\right\rfloor}^{1}\right)$ except that $T \cong S_{\left\lceil\frac{n-2}{2}\right\rceil,\left\lfloor\frac{n-2}{2}\right\rfloor}^{1}$.

Proof If $d(T)=3$, then $T \cong S_{a, b}^{1}$ for some positive integers $a, b$ with $a+b=n-2$. It is well known that the Laplacian characteristic polynomial of $S_{a, b}^{1}$ is $\mu(\mu-1)^{n-4} f_{a, b}(\mu)$, where

$$
f_{a, b}(\mu)=\mu^{3}-(n+2) \mu^{2}+(a b+2 n+1) \mu-n .
$$

Note that $S_{a, b}^{1}$ contains $S_{1,1}^{1}$ as a subtree. By Lemma 2.9, $\mu_{2}\left(S_{a, b}^{1}\right) \geq \mu_{2}\left(S_{1,1}^{1}\right)=2$. Moreover, we know that for any tree $T, \alpha(T) \leq 1$, with equality if and only if $T$ is a star. These imply that $\mu_{1}\left(S_{a, b}^{1}\right), \mu_{2}\left(S_{a, b}^{1}\right)$, and $\alpha\left(S_{a, b}^{1}\right)$ consist of the three roots of $f_{a, b}(\mu)$. As follows from (1), we have

$$
\mu_{1}\left(S_{a, b}^{1}\right)+\mu_{2}\left(S_{a, b}^{1}\right)+\alpha\left(S_{a, b}^{1}\right)=n+2 .
$$

By virtue of Lemma 2.6, we have $\alpha\left(S_{a, b}^{1}\right)>\alpha\left(S_{\left\lceil\frac{n-2}{2}\right\rceil,\left\lfloor\frac{n-2}{2}\right\rfloor}^{1}\right)$ except that $(a, b)=\left(\left\lceil\frac{n-2}{2}\right\rceil,\left\lfloor\frac{n-2}{2}\right\rfloor\right)$. Equivalently, $S\left(S_{a, b}^{1}\right)<S\left(S_{\left\lceil\frac{n-2}{2}\right\rceil,\left\lfloor\frac{n-2}{2}\right\rfloor}^{1}\right)$ except that $(a, b)=\left(\left\lceil\frac{n-2}{2}\right\rceil,\left\lfloor\frac{n-2}{2}\right\rfloor\right)$.

If $d(T)=2$, then $T \cong S_{n}$. We first give a lower bound of $S\left(S_{\left\lceil\frac{n-2}{2}\right\rceil,\left\lfloor\frac{n-2}{2}\right\rfloor}^{1}\right)$ for $n \geq 6$ :

$$
S\left(S_{\left\lceil\frac{n-2}{2}\right\rceil,\left\lfloor\frac{n-2}{2}\right\rfloor}^{1}\right)>n+1.5
$$

Indeed, by (2) it suffices to show $\alpha\left(S_{\left\lceil\frac{n-2}{2}\right\rceil,\left\lfloor\frac{n-2}{2}\right\rfloor}^{1}\right)<0.5$. Note that for $n \geq 6, S_{\left\lceil\frac{n-2}{2}\right\rceil,\left\lfloor\frac{n-2}{2}\right\rfloor}^{1}$ contains $S_{2,2}^{1}$ as a subtree. By Lemma 2.7, $\alpha\left(S_{\left\lceil\frac{n-2}{2}\right\rceil,\left\lfloor\frac{n-2}{2}\right\rfloor}^{1}\right) \leq \alpha\left(S_{2,2}^{1}\right)=\frac{5-\sqrt{17}}{2}<0.5$. 
Note that $S\left(S_{n}\right)=n+1$ for $n \geq 3$. According to (3), we have $S\left(S_{n}\right)<S\left(S_{\left\lceil\frac{n-2}{2}\right\rceil,\left\lfloor\frac{n-2}{2}\right\rfloor}^{1}\right)$ for $n \geq 6$. As for $n \in\{4,5\}$, a straightforward calculation shows that

$$
S\left(S_{1,1}^{1}\right) \approx 5.4142, \quad S\left(S_{2,1}^{1}\right) \approx 6.4811 .
$$

Also we have $S\left(S_{n}\right)<S\left(S_{\left\lceil\frac{n-2}{2}\right\rceil,\left\lfloor\frac{n-2}{2}\right\rfloor}^{1}\right)$.

Claim 2.12 For any tree $T$ with order $n$ and diameter $d \geq 5, S(T)<S\left(S_{\left\lceil\frac{n-2}{2}\right\rceil,\left\lfloor\frac{n-2}{2}\right\rfloor}^{1}\right)$.

Proof Since $d(T) \geq 5$, then $n \geq 6$ and there is a path of length 5 in $T$. By inequality (3), it suffices to show $S(T) \leq n+1.5$. First suppose that there is a path $v_{0} v_{1} \cdots v_{5}$ in $T$ such that either $\max \left\{d_{T}\left(v_{0}\right), d_{T}\left(v_{5}\right)\right\} \geq 2$ or $\max \left\{d_{T}\left(v_{2}\right), d_{T}\left(v_{3}\right)\right\} \geq 3$. Let $T_{1}, T_{2}$ be the two components of $T-v_{2} v_{3}$. Clearly, both $T_{1}$ and $T_{2}$ have at least two edges.

If $\mu_{1}, \mu_{2}$ of $T_{1} \cup T_{2}$ attain at the same component, say $T_{1}$, then by Lemma 2.2,

$$
S\left(T_{1} \cup T_{2}\right)=S\left(T_{1}\right) \leq m\left(T_{1}\right)+3 \leq m\left(T_{1} \cup T_{2}\right)+1 .
$$

Note that $S\left(v_{2} v_{3}\right)=S\left(S_{2}\right)=2$. By Lemma 2.1,

$$
S(T) \leq S\left(T_{1} \cup T_{2}\right)+S\left(v_{2} v_{3}\right) \leq m\left(T_{1} \cup T_{2}\right)+3=m(T)+2=n+1 .
$$

Otherwise, $S\left(T_{1} \cup T_{2}\right)=\mu_{1}\left(T_{1}\right)+\mu_{1}\left(T_{2}\right)$. Whether $\max \left\{d_{T}\left(v_{0}\right), d_{T}\left(v_{5}\right)\right\} \geq 2$ or $\max \left\{d_{T}\left(v_{2}\right), d_{T}\left(v_{3}\right)\right\} \geq 3$, we can observe that $\max \left\{d\left(T_{1}\right), d\left(T_{2}\right)\right\} \geq 3$. Say $d\left(T_{2}\right) \geq 3$, then by Corollary 2.5, $\mu_{1}\left(T_{2}\right)<n\left(T_{2}\right)-0.5$. By Lemma 2.3(ii), $\mu_{1}\left(T_{1}\right) \leq n\left(T_{1}\right)$. Hence,

$$
S(T) \leq S\left(T_{1} \cup T_{2}\right)+S\left(v_{2} v_{3}\right)=n\left(T_{1}\right)+n\left(T_{2}\right)-0.5+2=n+1.5 .
$$

Next, we may assume that each path $v_{0} v_{1} \cdots v_{5}$ of length 5 in $T$ has $d_{T}\left(v_{0}\right)=d_{T}\left(v_{5}\right)=1$ and $d_{T}\left(v_{2}\right)=d_{T}\left(v_{3}\right)=2$. This implies that $d(T)=5$ and $T \cong S_{a, b}^{3}$ for some integers $a, b$ with $a+b=n-4$. If $a=b=1$, then $T$ is isomorphic to a path of order 6 and a straightforward calculation shows that $S(T)=5+\sqrt{3}<n+1.5$, as claimed. Otherwise, assume without loss of generality that $a \geq 2$. Then $d_{T}\left(v_{1}\right) \geq 3$. Let $T_{3}, T_{4}$ be the two components of $T-v_{1} v_{2}$ with $v_{0} v_{1} \in E\left(T_{3}\right)$. Then both $T_{3}$ and $T_{4}$ have at least two edges. If $\mu_{1}, \mu_{2}$ of $T_{3} \cup T_{4}$ attain at the same component, say $T_{3}$, then by Lemmas 2.1 and 2.2,

$$
S(T) \leq S\left(T_{3} \cup T_{4}\right)+S\left(v_{1} v_{2}\right)=S\left(T_{3}\right)+2 \leq m\left(T_{3}\right)+5 \leq m(T)+2=n+1 .
$$

Otherwise, $S\left(T_{3} \cup T_{4}\right)=\mu_{1}\left(T_{3}\right)+\mu_{1}\left(T_{4}\right)$. Note that $\mu_{1}\left(T_{3}\right) \leq n\left(T_{3}\right)$. Since $d\left(T_{4}\right)=3$, by Corollary 2.5, $\mu_{1}\left(T_{4}\right)<n\left(T_{4}\right)-0.5$. So

$$
S(T) \leq S\left(T_{3} \cup T_{4}\right)+S\left(v_{1} v_{2}\right) \leq n\left(T_{3}\right)+n\left(T_{4}\right)-0.5+2=n+1.5 .
$$

Claim 2.13 For any tree $T$ with order $n$ and diameter $4, S(T)<S\left(S_{\left\lceil\frac{n-2}{2}\right\rceil,\left\lfloor\frac{n-2}{2}\right\rfloor}^{1}\right)$.

Proof First suppose that $T$ contains a path $v_{0} v_{1} \cdots v_{4}$ such that $\max \left\{d_{T}\left(v_{1}\right), d_{T}\left(v_{3}\right)\right\} \geq 3$. Now $n \geq 6$ and it suffices to show $S(T) \leq n+1.5$. Without loss of generality assume that 
$d_{T}\left(v_{1}\right) \geq 3$. Let $T_{1}, T_{2}$ be the two components of $T-v_{1} v_{2}$ with $v_{0} v_{1} \in E\left(T_{1}\right)$. Then both $T_{1}$ and $T_{2}$ have at least two edges.

If $\mu_{1}, \mu_{2}$ of $T_{1} \cup T_{2}$ attain at the same component, say $T_{1}$, then similarly to inequalities (5) and (6), we can observe that $S(T) \leq n+1$.

Now let $S\left(T_{1} \cup T_{2}\right)=\mu_{1}\left(T_{1}\right)+\mu_{1}\left(T_{2}\right)$. If $d_{T}\left(v_{2}\right) \geq 3$, then $d\left(T_{2}\right) \geq 3$ and hence $\mu_{1}\left(T_{2}\right)<$ $n\left(T_{2}\right)-0.5$. So

$$
S(T) \leq S\left(T_{1} \cup T_{2}\right)+S\left(v_{1} v_{2}\right)<n\left(T_{1}\right)+n\left(T_{2}\right)-0.5+2=n+1.5 .
$$

If $d_{T}\left(v_{2}\right)=2$, then $T \cong S_{a, b}^{2}$ for some positive integers $a, b$ with $3 \leq a+b=n-3$. Moreover, since $d_{T}\left(v_{1}\right) \geq 3$, then $a \geq 2$. If $(a, b) \in\{(2,1),(3,1)\}$, a straightforward calculations show that $S\left(S_{a, b}^{2}\right)<n+1.5$. Otherwise, $S_{a, b}^{2}$ contains either $S_{4,1}^{2}$ or $S_{2,2}^{2}$ as a subtree. Since

$$
\mu_{3}\left(S_{4,1}^{2}\right) \approx 1.5068, \quad \mu_{3}\left(S_{2,2}^{2}\right) \approx 1.5858,
$$

it follows from Lemma 2.9 that $\mu_{3}\left(S_{a, b}^{2}\right)>1.5$. Since $S_{a, b}^{2}$ is not a star, $\mu_{n-1}\left(S_{a, b}^{2}\right)<1$. On the other hand, note that the matrix $1 \cdot I_{n}-\left[D\left(S_{a, b}^{2}\right)-A\left(S_{a, b}^{2}\right)\right]$ has $a$ identical rows and $b$ different identical rows, so the multiplicity of eigenvalue 1 is at least $a+b-2$ and else five eigenvalues are $\mu_{1}, \mu_{2}, \mu_{3}, \mu_{n-1}$ and $\mu_{n}=0$. Since $\sum_{i=1}^{n} \mu_{i}\left(S_{a, b}^{2}\right)=2(n-1)$, we have

$$
\sum_{i=1}^{3} \mu_{i}\left(S_{a, b}^{2}\right)+\mu_{n-1}\left(S_{a, b}^{2}\right)+\mu_{n}\left(S_{a, b}^{2}\right)=2(n-1)-(a+b-2)=n+3 .
$$

This implies that $S\left(S_{a, b}^{2}\right)<n+3-\mu_{3}\left(S_{a, b}^{2}\right)<n+1.5$.

Next, it suffices to consider the case that each path $v_{0} v_{1} \cdots v_{4}$ of $T$ has $d_{T}\left(v_{1}\right)=d_{T}\left(v_{3}\right)=2$. This implies that $T \cong T_{n}^{k}$ for some $k \geq 2$ and $n \geq 2 k+1$, since $d(T)=4$. According to Lemma 2.8, $\mu_{2}\left(T_{n}^{k}\right)=\frac{3+\sqrt{5}}{2}$. Moreover, by Lemma 2.3,

$$
\mu_{1}\left(T_{n}^{k}\right) \leq \max \left\{d_{i}+m_{i}\right\}=n-k-1+\frac{n-1}{n-k-1} \leq n-2+\frac{2}{n-3} .
$$

Thus for $n \geq 6$,

$$
S\left(T_{n}^{k}\right) \leq n-2+\frac{2}{n-3}+\frac{3+\sqrt{5}}{2}<n+1.5<S\left(S_{\left\lceil\frac{n-2}{2}\right\rceil,\left\lfloor\frac{n-2}{2}\right\rfloor}^{1}\right) .
$$

When $n=5, T_{n}^{k}$ is a path. Comparing with (4), $S\left(T_{n}^{k}\right)=4+\sqrt{5}<S\left(S_{2,1}^{1}\right)$. This completes the proof.

Following from Claims 2.11-2.13, Theorem 2.10 holds and the unique tree with maximal $S(T)$ is $S_{\left\lceil\frac{n-2}{2}\right\rceil,\left\lfloor\frac{n-2}{2}\right\rfloor}^{1}$. According to (2),

$$
\mu\left(S_{\left\lceil\frac{n-2}{2}\right\rceil,\left\lfloor\frac{n-2}{2}\right\rfloor}^{1}\right)<n+2=m\left(S_{\left\lceil\frac{n-2}{2}\right\rceil,\left\lfloor\frac{n-2}{2}\right\rfloor}^{1}\right)+3 .
$$

Theorem 2.14 Let $m, n$ be two positive integers with $n \leq m \leq 2 n-3$ and $G_{m, n}$ be a graph of order $n$ and size $m$ obtained from a given edge uv by joining $m-n+1$ independent vertices with $u$ and $v$, respectively, and another $2 n-m-3$ independent vertices with $u$. Then $S\left(G_{m, n}\right)=m+3$. 
Proof Let $H_{s, t}$ be a graph obtained by joining a vertex to $s$ vertices of a given complete graph of order $s+t$ and $H_{s, t}^{c}$ be its complement graph. Then $H_{s, t}^{c}$ is isomorphic to the union of $S_{t+1}$ and $s$ isolated vertices. Clearly, the Laplacian eigenvalues of $H_{s, t}^{c}$ consist of $t+1,1$ with multiplicity $t-1$ and 0 with multiplicity $s+1$. Recall that for any graph $G$ with $n$ vertices, $\mu_{i}(G)=n-\mu_{n-i}\left(G^{c}\right)$ for $1 \leq i \leq n-1$ and $\mu_{n}(G)=0$. So the Laplacian eigenvalues of $H_{s, t}$ consist of $s+t+1$ with multiplicity $s, s+t$ with multiplicity $t-1, s$ and 0 .

Now $G_{m, n}^{c}$ is isomorphic to the union of $H_{2 n-m-3, m-n+1}$ and an isolated vertex. So the Laplacian eigenvalues of $G_{m, n}^{c}$ consist of $n-1$ with multiplicity $2 n-m-3, n-2$ with multiplicity $m-n, 2 n-m-3$, and 0 with multiplicity 2 . Therefore, the Laplacian eigenvalues of $G_{m, n}$ consist of $n, m-n+3,2$ with multiplicity $m-n, 1$ with multiplicity $2 n-m-3$ and 0 . So $S\left(G_{m, n}\right)=n+(m-n+3)=m+3$.

Recall that $\mu_{1}(G) \leq n(G)$ for any graph $G$. When $m(G)>2 n(G)-3$, Haemers' bound is clearly not attainable. Theorem 2.14 implies that if $m(G) \leq 2 n(G)-3$, Haemers' bound is always sharp for connected graphs other than trees. Ending the paper, we present a conjecture on the uniqueness of the extremal graph.

Conjecture 2.15 Among all connected graphs with $n$ vertices and $n \leq m \leq 2 n-3$ edges, $G_{m, n}$ is the unique graph with maximal value of $\mu_{1}+\mu_{2}$.

\section{Competing interests}

The authors declare that they have no competing interests.

\section{Authors' contributions}

MG carried out the proofs of the main results in the manuscript. MQZ and YFW participated in the design of the study and drafted the manuscript. All authors read and approved the final manuscript.

\section{Author details}

'Department of Mathematics and Physics, Hefei University, Hefei, 230601, China. ${ }^{2}$ School of Mathematical Science, Chuzhou University, Chuzhou, 239012, China. ${ }^{3}$ College of Mathematics and Computer Science, Tongling University, Tongling, 244000, China.

\section{Acknowledgements}

The authors are grateful to the referees for carefully reading the manuscript and for providing some comments and suggestions, which led to improvements in the paper. The research was supported by the National Natural Science Foundation of China (11101057, 11201432), the Foundation for Young Talents in College of Anhui Province (2012SQRL170) and the Natural Science Foundation of Anhui Province (1308085MA03).

Received: 23 December 2013 Accepted: 5 June 2014 Published: 23 June 2014

\section{References}

1. Bıyıkoğlu, T, Leydold, J: Algebraic connectivity and degree sequences of trees. Linear Algebra Appl. 430, 811-817 (2009)

2. Fallat, S, Kirkland, S: Extremizing algebraic connectivity subject to graph theoretic constraints. Electron. J. Linear Algebra 3, 48-74 (1998)

3. Fan, YZ, Xu, J, Wang, Y, Liang, D: The Laplacian spread of a tree. Discrete Math. Theor. Comput. Sci. 10(1), 79-86 (2008)

4. Guo, JM: On the second largest Laplacian eigenvalue of trees. Linear Algebra Appl. 404, 251-261 (2005)

5. Shao, JY, Zhang, L, Yuan, XY: On the second Laplacian eigenvalues of trees of odd order. Linear Algebra Appl. 419, 475-485 (2006)

6. Zhang, XD, Li, JS: The two largest eigenvalues of Laplacian matrices of trees. J. Univ. Sci. Technol. China 28, 513-518 (1998)

7. Anderson, WN, Morley, TD: Eigenvalues of the Laplacian of a graph. Linear Multilinear Algebra 18, 141-145 (1985)

8. Haemers, WH, Mohammadian, A, Tayfeh-Rezaie, B: On the sum of Laplacian eigenvalues of graphs. Linear Algebra Appl. 432, 2214-2221 (2010)

9. Brouwer, AE, Haemers, WH: Spectra of Graphs. Springer, New York (2012)

10. Fritscher, E, Hoppen, C, Rocha, I, Trevisan, V: On the sum of the Laplacian eigenvalues of a tree. Linear Algebra Appl. 435, 371-399 (2011)

11. Grone, R, Merris, R, Sunder, VS: The Laplacian spectrum of a graph II. SIAM J. Matrix Anal. Appl. 11, 218-238 (1990)

12. Merris, R: A note on the Laplacian graph eigenvalues. Linear Algebra Appl. 285, $33-35$ (1998)

13. Godsil, C, Royle, G: Algebraic Graph Theory. Springer, New York (2001) 
doi:10.1186/1029-242X-2014-242

Cite this article as: Guan et al.: On the sum of the two largest Laplacian eigenvalues of trees. Journal of Inequalities and Applications 2014 2014:242.

Submit your manuscript to a SpringerOpen ${ }^{\circ}$ journal and benefit from:

- Convenient online submission

- Rigorous peer review

- Immediate publication on acceptance

Open access: articles freely available online

- High visibility within the field

- Retaining the copyright to your article

Submit your next manuscript at $\boldsymbol{s p r i n g e r o p e n . c o m ~}$ 